\title{
Nueva especie de Tityus (Scorpiones: Buthidae) del Turimiquire, Venezuela
}

\author{
Leonardo De Sousa ${ }^{1}$, Jesús Manzanilla ${ }^{2}$ \& Pedro Parrilla-Álvarez ${ }^{3}$ \\ 1 Centro de Investigaciones en Ciencias de la Salud (CICS), Escuela de Medicina, Universidad de Oriente, Núcleo de \\ Anzoátegui, Apartado Postal 4774, Puerto La Cruz 6023, Estado Anzoátegui, Venezuela' leonardodesousa@yahoo.com \\ 2 Instituto de Zoología Agrícola, Facultad de Agronomía, Universidad Central de Venezuela, Apartado 4579, Maracay \\ 2101-A, Estado Aragua, Venezuela; jesusmanzanillap@hotmail.com \\ 3 Laboratorio de Alacranología, Escuela de Medicina, Universidad de Oriente, Núcleo de Bolívar, Ciudad Bolívar, \\ Estado Bolívar, Venezuela.
}

Recibido 20-II-2003. Corregido 11-XI-2003. Aceptado 16-IV-2004.

\begin{abstract}
A new species of Tityus (Scorpiones: Buthidae) from Turimiquire, Venezuela. We describe a new species of Buthid scorpion, Tityus quirogae, from the Turimiquire mountains, eastern side of the La Costa range, Monagas and Sucre States, Venezuela. It is morphologically similar to T. nematochirus and T. meridanus. It differs from these species in the distribution of the dorsal femoral trichobothria and in the following combination of characters: (1) I caudal segment with two parallel ventral keels. Caudal segments II to IV with ventral keels that are double in the extremes of the segments and single in the middle, (2) noticeable sexual dimorphism, especially in pedipalp shape; the male chelae are markedly thin and long, (3) number of pectineous teeth (male: 18 in each pecten; female: 18 in the right pecten and 19 in the left one), and (4) number of denticle rows in the movable finger of the right chela (16 in both sexes). T. quirogae is the first species of this genus, in the Northeastern region of Venezuela, with thin and elongated pedipalps. Rev. Biol. Trop. 54(2): 489-504. Epub 2006 Jun 01.
\end{abstract}

Key words: scorpion, Buthidae, Tityus, new species, Turimiquire, Venezuela.

En Venezuela la familia Buthidae está representada por siete géneros (De Sousa et al. 2000). Las especies de Tityus conocidas hasta el año 2004 ascienden a 44 (GonzálezSponga 1996a, b, 1997a, b, 2001, De Sousa et al. 2000, Quiroga et al. 2000, 2004). Estas han sido agrupadas en tres "grupos artificiales" denominados: "discrepans", "nematochirus" y "androcottoides" (González-Sponga 1996a). El grupo "nematochirus" incluye a T. nematochirus (Mello-Leitão 1940, 1945) y T. meridanus (González-Sponga 1981). Este grupo se caracteriza por presentar carenas ventrales dobles y completas en los segmentos caudales I al IV del metasoma. T. nematochirus y T. meridanus representan una excepción del género, en Venezuela, porque sus pedipalpos y particularmente las quelas son estrechos en los ejemplares machos (González-Sponga 1984). Estos escorpiones con pedipalpos delgados sólo se han encontrado en los estados andinos (Táchira y Mérida) ubicados en la región occidental del país (González-Sponga 1996a).

Para los estados Monagas y Sucre se han señalado siete especies de Tityus (De Sousa et al. 2000, González-Sponga 2001), una de ellas pertenece al grupo "discrepans" (T. arellanoparrai, González-Sponga 1985), una al grupo "nematochirus" (T. tamayoi, González-Sponga 1987) y cuatro al grupo "androcottoides" (T. monaguensis, González-Sponga 1974; T. surorientalis, GonzálezSponga 1996b; T. nororientalis, González-Sponga 1996b; T. caripitensis, Quiroga et al. 2000 y T. uquirensis, González-Sponga 2001).

En este trabajo se describe una nueva especie de Tityus de quelas delgadas y largas, 
capturada en la región Nororiental de Venezuela. Morfológicamente es similar a T. nematochirus y a $T$. meridanus, dos especies con dimorfismo sexual en los pedipalpos. El nuevo taxón representa la novena especie de Tityus para el nororiente venezolano. Este nuevo hallazgo refuerza los criterios que determinan la presencia de una región endémica para el escorpionismo en los estados Anzoátegui, Monagas y Sucre (De Sousa et al. 1999, 2000) y contribuye con la elaboración del mapa de distribución del escorpionismo provocado por las especies de Tityus en Venezuela (Borges 1996).

\section{MATERIALES Y MÉTODOS}

Material examinado. Los ejemplares fueron obtenidos, entre los años 1998 y 2002, por captura activa en sus refugios naturales. Durante el día fueron localizados bajo troncos, en el interior de la corteza parcialmente desprendida de los árboles, en el interior de troncos en proceso de descomposición y en la base de las plantas de cafeto. Durante la noche se utilizaron lámparas portátiles de luz ultravioleta para ubicarlos en el talud de la carretera. Los especimenes se encuentran depositados en la Colección de Escorpiones del Laboratorio de Toxinología (CELT), Centro de Investigaciones en Ciencias de la Salud (CICS), Escuela de Medicina, Universidad de Oriente (UDO), Núcleo de Anzoátegui, Venezuela y en la Colección de Escorpiones del Museo del Instituto de Zoología Agrícola (MIZA), Facultad de Agronomía, Universidad Central de Venezuela (UCV), Venezuela.

Se conformó una Serie Tipo (constituida por 10 ejemplares: 1 holotipo macho, 4 paratipos machos y 5 paratipos hembras) y se estableció una Serie Morfométrica (17 machos y 31 hembras; que incluye a la Serie Tipo) para obtener las medianas según el método de Hodges y Lehmann y sus límites de confianza al $95 \%$ de certeza para establecer las variaciones de la especie y el dimorfismo sexual entre machos y hembras. La diferencia entre las medianas fue probado por la técnica de análisis de variancia de Kruskal-Wallis, con un nivel de significación $\mathrm{p}<0.05$. Para el procesamiento de los datos se utilizó el paquete estadístico V-8.2 del Dr. Carlos Sevcik, Laboratorio de Neurofarmacología Celular, Centro de Biofísica y Bioquímica, IVIC, Caracas.

Se utilizó como base para el estudio de las tricobotrias la nomenclatura propuesta por Vachon (1974) y para las estructuras anatómicas la de Stahnke (1970) y Manzanilla et al. (2002). Los caracteres morfológicos medidos fueron: (1) longitud y ancho del caparazón, (2) longitud de la quela izquierda y derecha, (3) ancho de la quela derecha, (4) longitud del dedo móvil de la quela derecha, (5) longitud de la tibia derecha, (6) longitud del fémur derecho, (7) longitud del primer al quinto segmento caudal, (8) ancho del primer al quinto segmento caudal, (9) longitud del telson, (10) longitud del peine derecho y (11) longitud total del escorpión. Las mediciones se presentan en milímetros ( $\mathrm{mm}$ ) y se tomaron bajo microscopio estereoscópico con un vernier digital (Mitutoyo ${ }^{\circledR}$ ). Las ilustraciones fueron elaboradas con un microscopio estereoscópico $\left(\right.$ Leyka $\left.^{\circledR}\right)$ provisto de cámara lúcida.

\section{RESULTADOS}

\section{Tityus quirogae sp. nov.}

Figs. 1-5

Holotipo. CELT-337, macho adulto, proveniente de la hacienda cafetalera La Encantada (10¹4'06" N y 6332'00" W, 1172 metros sobre el nivel del mar: GPS 45 Garmin $^{\circledR}$ ) sector Vuelta Larga de la población de Sabana de Piedra, Municipio Caripe, Estado Monagas, Venezuela. Colector: Alcides Betancourt, 20/iii/2000.

Paratipos. Cuatro machos adultos; CELT320: A. Betancourt, 24/x/1999, hacienda cafetalera La Encantada, sector Vuelta Larga, Sabana de Piedra, Municipio Caripe, Estado Monagas, Venezuela. CELT-336: A. Betancourt, 20/ iii/2000, hacienda cafetalera La Encantada, sector Vuelta Larga, Sabana de Piedra, Municipio 
Caripe. CELT-363: E. Asens, 17/xi/2000, La Guanota, Municipio Caripe. CELT-400: E. Asens, 22/ii/2001, La Guanota, Municipio Caripe. Cinco hembras adultas; CELT-152: L. De Sousa, 27/iii/1998, sector La Cajuira, caserío La Loma, Las Trincheras, Municipio Montes, Estado Sucre. CELT-246: A. Betancourt, 17/ ix/1999, hacienda cafetalera Tacarigua, sector Palma Sola, Sabana de Piedra, Municipio Caripe. CELT-323: A. Betancourt, 30/x/1999, hacienda cafetalera Palma Sola, sector Palma Sola, Sabana de Piedra, Municipio Caripe. CELT-334: E. Asens, 23/ii/2000, La Guanota, Municipio Caripe. CELT-399: E. Asens, 22/ii/2001, La Guanota, Municipio Caripe.

Definición y diagnosis. T. quirogae se diferencia de las especies afines por la combinación de las siguientes características: (1) I segmento caudal con dos carenas ventrales paralelas, (2) segmentos caudales II y III con carenas ventrales dobles que convergen y finalmente divergen hacia la región distal, (3) IV segmento con dos hileras medianas paralelas en el quinto anterior, fusionadas en el resto del segmento; divergen en la región distal formando una "v", (4) por el número de dientes pectíneos del peine derecho: macho 18; hembra 18, (5) por el número de hileras de dentículos del dedo móvil de la quela derecha: macho 16 y hembra 16, (6) por presentar marcado dimorfismo sexual, especialmente en la forma de los pedipalpos: machos con quelas marcadamente delgadas y largas.

Se diferencia de $T$. nematochirus y T. meridanus, las dos especies más similares morfológicamente, pero geográficamente lejanas, en la disposición de las carenas ventrales de los segmentos caudales II al IV (en estas especies son dobles y completas) y por la distribución de las tricobotrias dorsales del fémur $\left(d_{3}\right.$ a $d_{5}$ ), las cuales se orientan en línea recta en $T$. quirogae. Se asemeja a T. valerae Scorza, 1954 del grupo "androcottoides" sensu GonzálezSponga (1996a) en la forma y disposición de las carenas de los segmentos I al V del metasoma y en el telson. Se diferencia por la longitud total, siendo mayor en T. quirogae (macho: $80.49 \mathrm{~mm}$; hembra: $81.04 \mathrm{~mm}$ ) que en T. valerae (macho: $58.20 \mathrm{~mm}$; hembra: $73.88 \mathrm{~mm}$ ), y en la forma notablemente diferente de los pedipalpos en los machos de T. quirogae (delgados en $T$. quirogae y globosos en T. valerae).

\section{ENGLISH DESCRIPTION}

The new species is morphologically similar to T. nematochirus and T. meridanus, but it differs from these species in the distribution of the dorsal trichobothria of the femur and in the following combination of features: (1) I caudal segment with two parallel caudal keels. Caudal segments II to IV with ventral keels that are double in the extremes of the segments and single in the middle, (2) noticeable sexual dimorphism, especially in the pedipalp shape; the chelae in the males are markedly thin and long, (3) number of pectineous teeth (male: 18 in each pecten; female: 18 in the right pecten and 19 in the left one), (4) number of rows of denticles in the movable finger of the right chela (16 in both sexes). T. quirogae is the first species of this genus, in the Northeastern region of Venezuela, with thin and elongated pedipalps.

\section{Descripción del holotipo macho}

Figs. 1b, 1c

Caparazón. (Fig. 2a). Borde anterior y posterior subparalelos, anterior ligeramente escotado formando ángulo obtuso. Bordes laterales con crestas continuas. Carenas paramedianas posteriores bien definidas, elevadas con gránulos planos. Carenas paramedianas anteriores formadas por hileras de gránulos espaciados y bien definidos. Carenas supraciliares bien definidas con gránulos próximos. Surco mediano-anterior, ancho y llano con pequeños gránulos dispersos. Surco interocular liso y acentuado. Surco mediano-posterior muy profundo con pocos gránulos bien definidos. Surcos postero-marginales acentuados. Surcos medianos-laterales poco acentuados. 

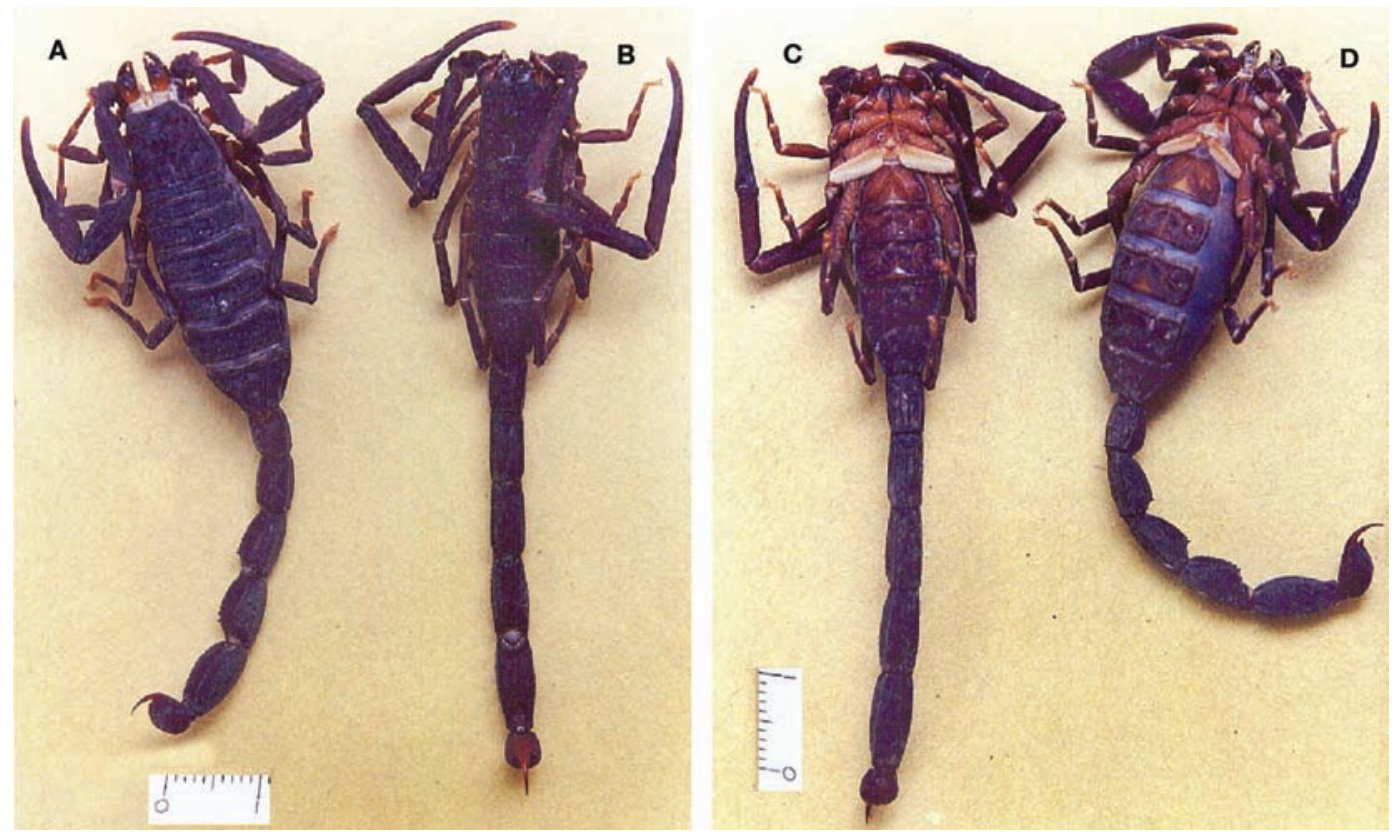

Fig. 1. Tityus quirogae sp. nov. Hembra (Paratipo, CELT-334): a. Vista dorsal. d. Vista ventral. Macho (Holotipo, CELT-337): b. Vista dorsal. c. Vista ventral. Escala $=1 \mathrm{~cm}$.

Fig. 1. Tityus quirogae sp. nov. Female (Paratype, CELT-334): a. Dorsal view. d. Ventral view. Male (Holotype, CELT-337): b. Dorsal view. c. Ventral view. Scale bar $=1 \mathrm{~cm}$.

Pedipalpos. (Figs. 2b, 2c). Segmentos muy largos y estrechos. Tegumento casi liso. Crestas poco acentuadas en mano, fémur y trocánter, con gránulos muy pequeños. Tibia con crestas continuas y gránulos no definidos. Presenta la tricobotriotaxia típica del género (Tipo A básico) (Sissom 1990). Tricobotrias dorsales $d_{3}$ a $d_{5}$ del fémur orientadas en línea recta, formando ángulo obtuso con la $d_{1}$ (Fig. 6e). Carenas externas, de la mano, vestigiales con filas de gránulos muy pequeños y espaciados. Las carenas dorsales y la digital son vestigiales. La mediana externa ocupa toda la quela. El dedo móvil presenta 16 hileras oblicuas de dentículos. Proporción ancho/largo de la quela $2.07 \mathrm{~mm} / 20.95 \mathrm{~mm}=0.1$. Proporción largo del dedo móvil/largo de la mano $12.70 \mathrm{~mm} /$ $20.95 \mathrm{~mm}=0.61$.

Patas. Tegumento liso, segmentos con crestas bajas y gránulos planos, escasos y espaciados.
Tergitos I al IV. Tegumento rugoso, con una carena mediana alta, bien definida. Cada tergito presenta una carena posterior formada por gránulos bien definidos. Una carena formada por gránulos a cada lado de las carenas medianas, las cuales se extienden próximas a los bordes laterales.

Esternitos I al IV. Una cresta mediana plana. Esternito IV con dos crestas paramedianas y esternito $\mathrm{V}$ con dos crestas medianas y dos paramedianas. Tegumento progresivamente rugoso desde el esternito I al V.

Esternón. (Fig. 2d). Subtriangular con surco profundo.

Peines. (Fig. 2d). Lamelas y fulcros bien definidos, cada uno con 18 dientes.

Segmentos caudales I al V. (Figs. 3a, 3b). Carenas bien definidas por gránulos de 

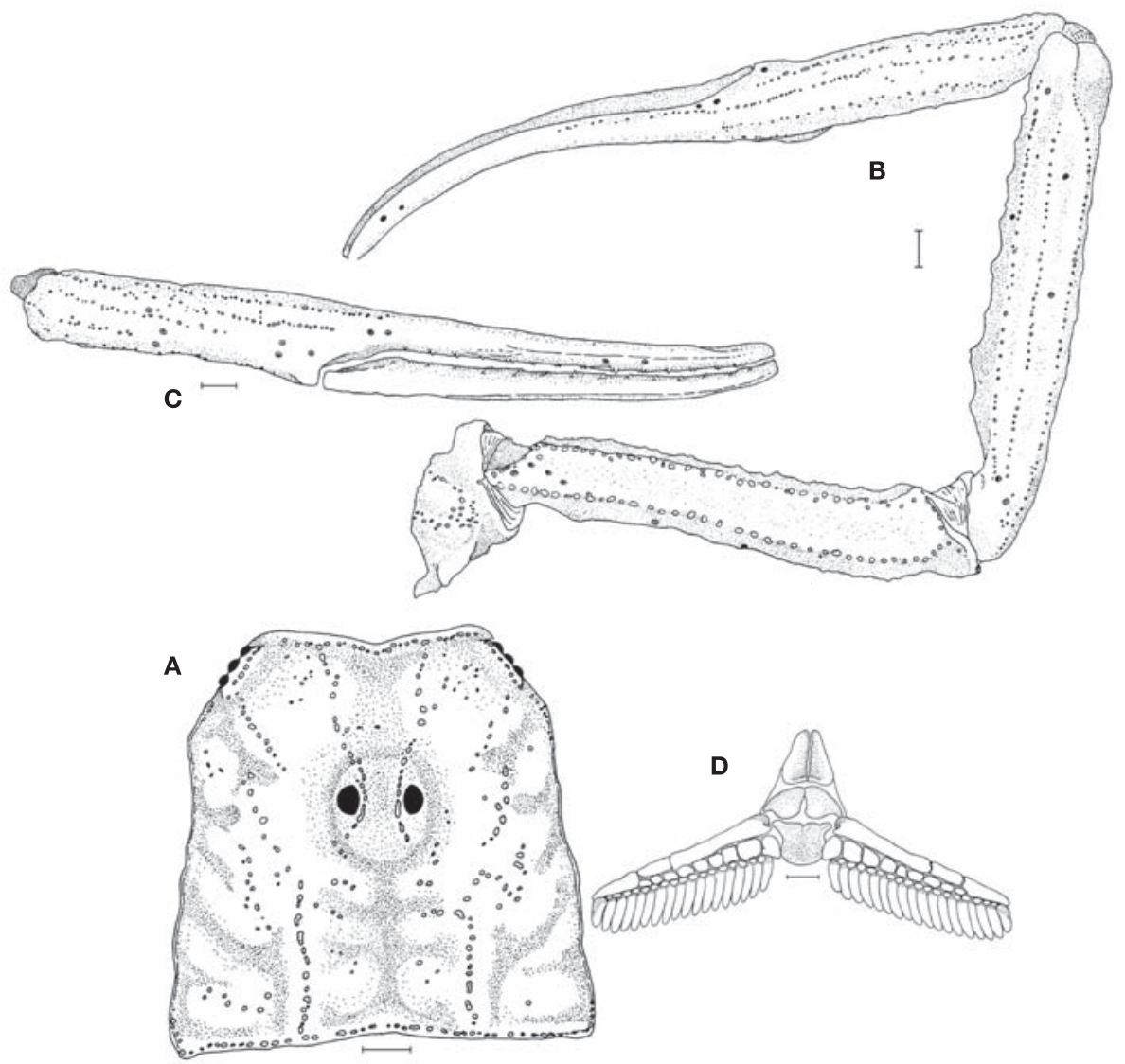

Fig. 2. Tityus quirogae sp. nov. Macho (CELT-337): a. Caparazón. b. Pedipalpo derecho, vista dorsal. c. Quela derecha, vista lateral externa. d. Esternón, opérculo genital y peines. Escala $=1 \mathrm{~mm}$.

Fig. 2. Tityus quirogae sp. nov. Male (CELT-337): a. Carapace. b. Rigth pedipalp, dorsal view. c. Rigth chela, external lateral view. d. Sternum, genital operculum and pectens. Scale bar $=1 \mathrm{~mm}$.

mediano tamaño alineados de manera regular. Segmentos I al IV con carenas mediodorsales, dorsolaterales y ventrales bien definidas. Segmento V con carena dorsolateral y lateroventral bien definidas aunque con gránulos planos. Gránulos de carenas dorsales bien definidos en todos los segmentos; terminan en un gránulo grande y espiniforme (Fig. 3b). Carenas ventrales (Fig. 3a) del I segmento con hileras medianas dobles y paralelas en toda su extensión. Segmento II con dos hileras medianas paralelas que se fusionan en el tercio distal para luego separarse nuevamente al término del segmento. Segmento III con dos hileras medianas paralelas en el primer tercio que se fusionan y divergen finalmente al término del segmento formando una "v". Segmento IV con dos hileras medianas paralelas en el quinto anterior, fusionadas en el resto del segmento; divergen en la región distal formando una " $v$ ". Segmento $\mathrm{V}$ con una carena mediana única en toda su extensión.

Telson. (Fig. 3c). Subovoide con hileras de gránulos formando crestas levemente definidas. Una cresta medioventral, dos lateroventrales y, a cada lado, una ventrolateral y una laterodorsal. Tubérculo subaculear mediano y puntiagudo, aproximadamente un quinto de la longitud del acúleo. 
Longitud total. $80.49 \mathrm{~mm}$. Las medidas del Holotipo se presentan en el Cuadro 1.

Coloración. (Figs. 1b, 1c). Castaño muy oscuro, casi negro en todo el cuerpo, excepto la región ventral, especialmente en la porción anterior correspondiente a las coxas de las patas, donde es castaño claro. Peines de color amarillo cremoso. La coloración de los adultos vivos es similar a la de los ejemplares preservados.

\section{Descripción del paratipo hembra \\ (CELT-334)}

Figs. 1a, 1d

Caparazón. (Fig. 4a). Borde anterior y posterior subparalelos, anterior ligeramente escotado formando ángulo obtuso. Bordes laterales con crestas continuas. Carenas paramedianas posteriores bien definidas, elevadas con gránulos planos. Carenas paramedianas

CUADRO 1

Serie Tipo: Datos morfométricos del holotipo y paratipos machos de Tityus quirogae

TABLE 1

Type Series: Morphometric data of the holoype and paratypes of Tityus quirogae

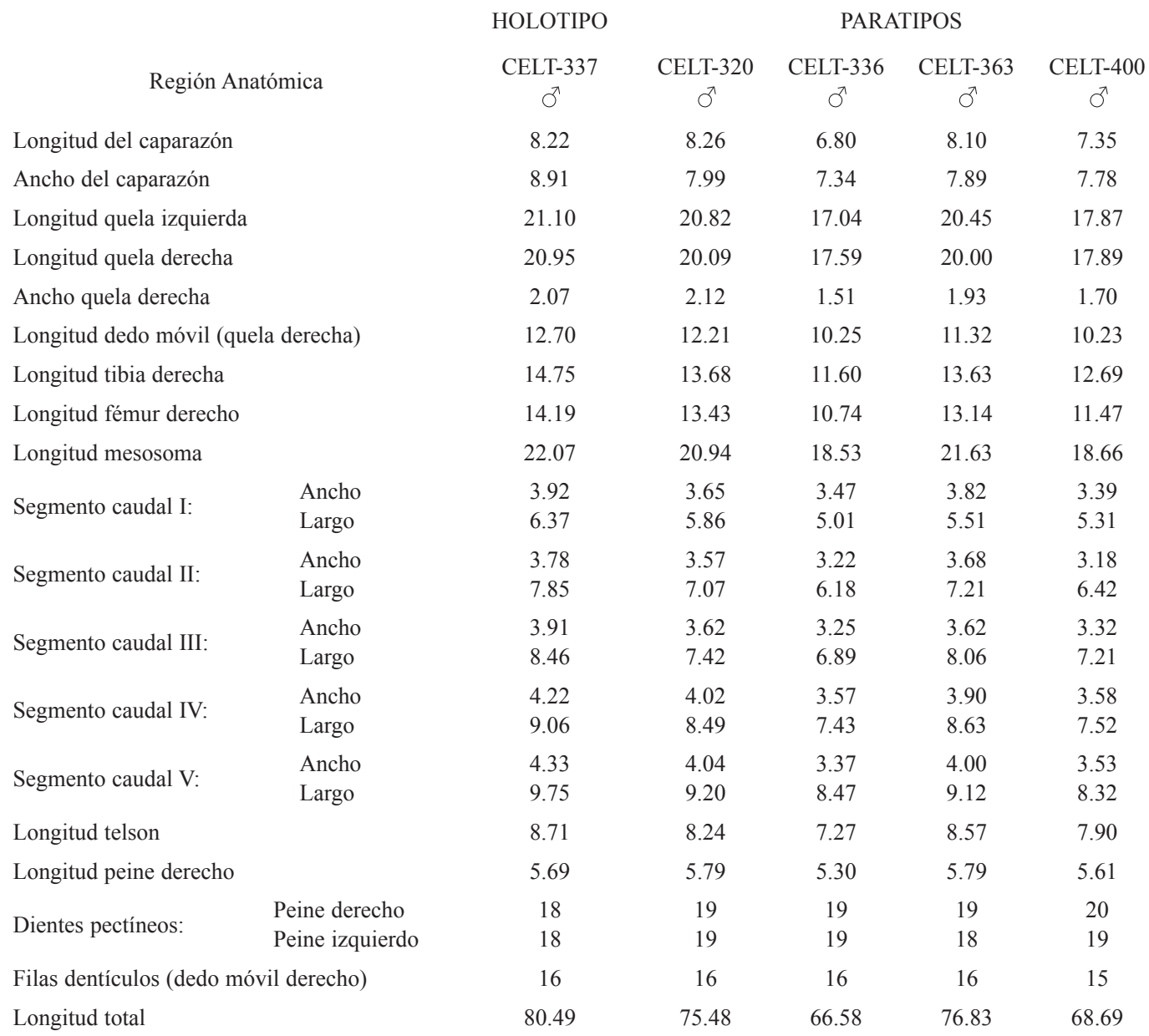

Todos los datos se expresan en mm (excepto el número de dientes pectíneos y el número de filas de dentículos del dedo móvil derecho). 


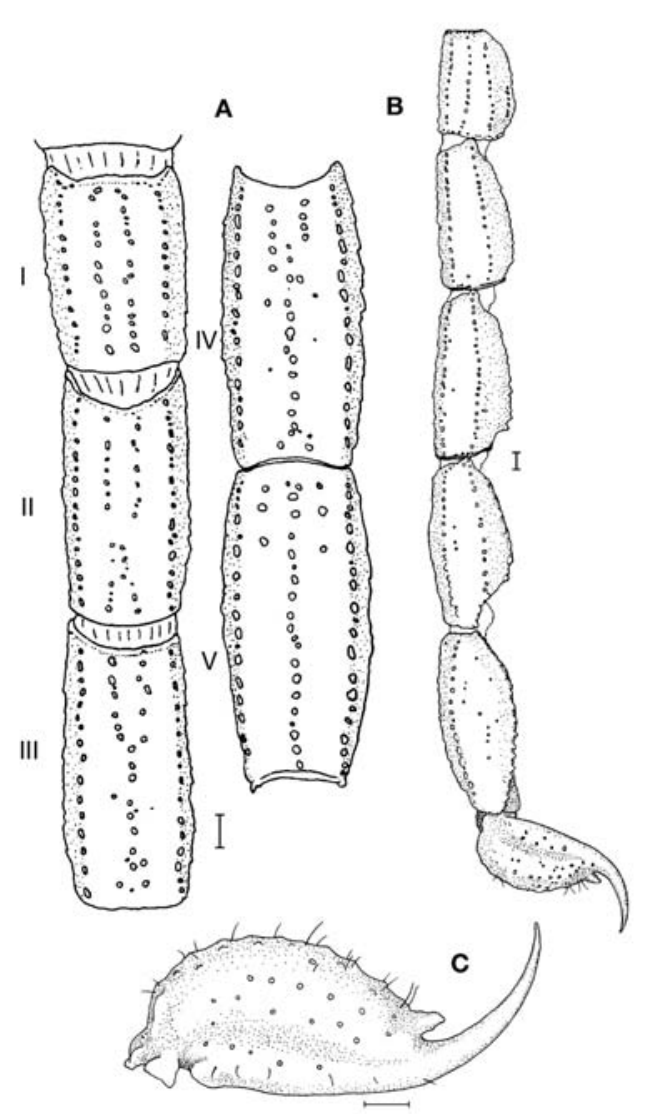

Fig. 3. Tityus quirogae sp. nov. Macho (CELT-337): a. Metasoma, vista ventral. b. Metasoma, vista lateral. c. Telson, vista lateral. Escala $=1 \mathrm{~mm}$.

Fig. 3. Tityus quirogae sp. nov. Male (CELT-337): a. Metasoma, ventral view. b. Metasoma, lateral view. c. Telson, lateral view. Scale bar $=1 \mathrm{~mm}$.

anteriores formadas por hileras de gránulos espaciados y bien definidos. Carenas supraciliares con gránulos poco definidos. Surco mediano-anterior, ancho y llano con pequeños gránulos dispersos. Surco interocular liso y acentuado. Surco mediano-posterior muy profundo con pocos gránulos bien definidos. Surcos postero-marginales acentuados. Surcos medianos-laterales poco acentuados.

Pedipalpos. (Figs. 4b, 4c). Segmentos menos largos y estrechos. Tegumento coriáceo casi liso. Crestas poco acentuadas en mano, fémur y trocánter, con gránulos muy pequeños. Tibia y fémur con crestas continuas y gránulos no definidos, exceptuando las ventrolaterales internas que muestran tubérculos espiniformes. Tricobotriotaxia típica del género (Tipo A básico) (Sissom 1990). Tricobotrias dorsales $d_{3}$ a $d_{5}$ del fémur orientadas en línea recta, formando ángulo recto con la $d_{1}$ (Fig. 6f). Carenas externas de la mano vestigiales, con filas de gránulos muy pequeños y espaciados. Las dorsales y digital también vestigiales. La mediana externa ocupa toda la mano. Dedo móvil con 16 hileras de dentículos. Proporción ancho/largo de la mano $3.00 \mathrm{~mm} / 16.09 \mathrm{~mm}=0.2$. Proporción largo del dedo móvil/largo de la mano 10.34 $\mathrm{mm} / 16.09 \mathrm{~mm}=0.64$.

Patas. Tegumento liso, segmentos con crestas bajas y gránulos planos, escasos y espaciados.

Tergitos I al IV. Tegumento rugoso, con carena mediana alta, bien definida. Cada tergito presenta una carena posterior formada por gránulos bien definidos. Una carena formada por gránulos a cada lado de las carenas medianas, las cuales se extienden próximas a los bordes laterales.

Esternitos I al IV. Con una cresta mediana plana. Esternito IV con dos crestas paramedianas y esternito $\mathrm{V}$ con dos crestas medianas y dos paramedianas. Tegumento progresivamente rugoso desde el primero hasta el quinto.

Esternón. Subtriangular con surco profundo.

Peines. (Fig. 4d). Lamelas y fulcros bien definidos, el derecho con 18 dientes y el izquierdo con 19.

Segmentos caudales I al V. (Figs. 5a, 5b). Carenas bien definidas conformadas por gránulos de mediano tamaño alineados de manera regular. Segmento I al IV con carenas mediodorsales, dorsolaterales y ventrales bien definidas. Segmento V con carena dorsolateral y lateroventral bien definidas aunque con 


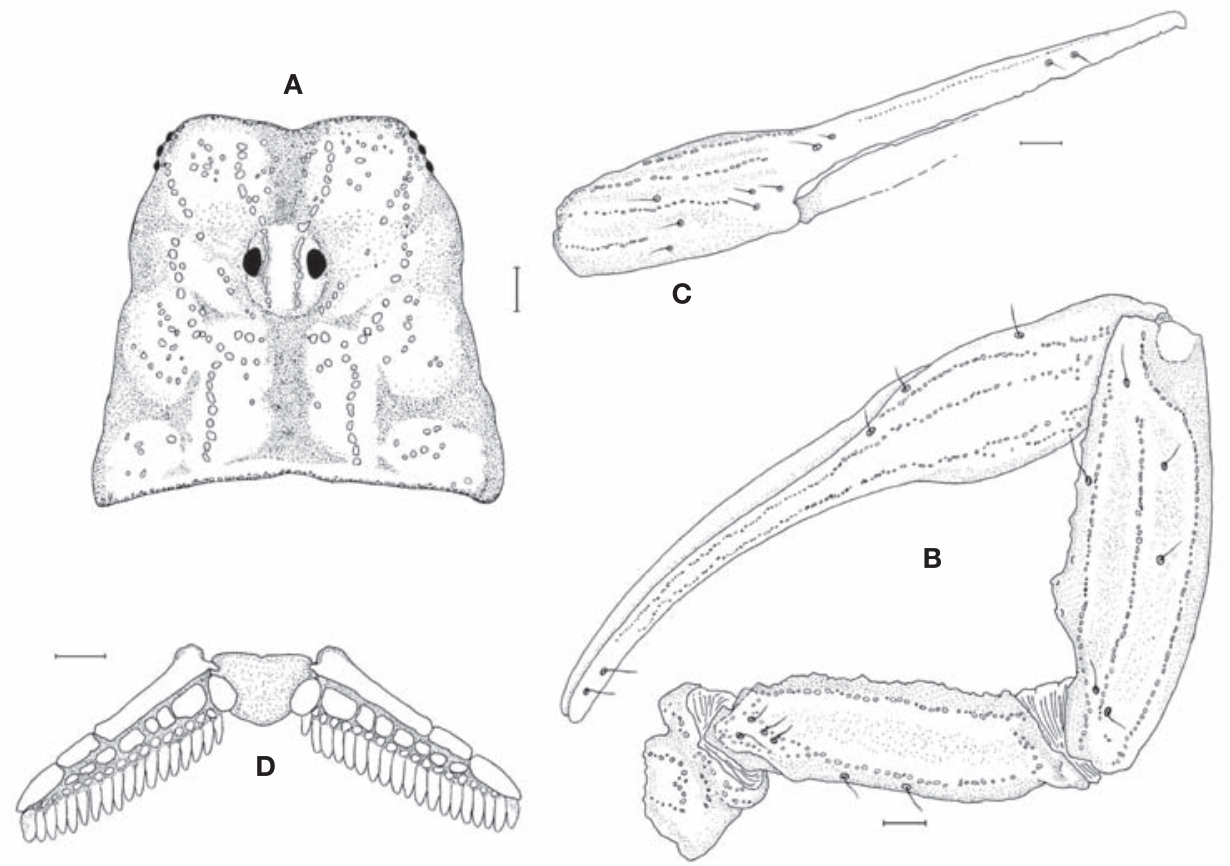

Fig. 4. Tityus quirogae sp. nov. Hembra (CELT-334): a. Caparazón. b. Pedipalpo derecho, vista dorsal. c. Quela derecha, vista lateral externa. d. Peines. Escala $=1 \mathrm{~mm}$.

Fig. 4. Tityus quirogae sp. nov. Female (CELT-334): a. Carapace. b. Rigth pedipalp, dorsal view. c. Rigth chela, external lateral view. d. Pectens. Scale bar $=1 \mathrm{~mm}$.

gránulos planos. Gránulos de las carenas dorsales bien definidos en todos los segmentos; terminan en un gránulo grande y espiniforme (Fig. 5b). Carenas ventrales (Fig. 5a) del segmento I con hileras medianas dobles y paralelas en toda su extensión. Segmento II con dos hileras medianas paralelas que se fusionan en el tercio distal para luego diverger nuevamente al término del segmento. Segmento III con dos hileras medianas paralelas en el primer tercio que se fusionan y divergen finalmente al final del segmento formando una "v". Segmento IV con dos hileras medianas paralelas en el quinto anterior, fusionadas en el resto del segmento; divergen en la región distal formando una "v". Segmento V con carena mediana única en toda su extensión.
Telson. (Fig. 5c). Subovoide con gránulos dispersos. Sólo la hilera ventral bien definida.

Longitud total. $81.04 \mathrm{~mm}$. En los Cuadros 1 y 2 se presentan los valores de la Serie Tipo.

Coloración. Castaño muy oscuro, casi negro en todo el cuerpo, exceptuando la región ventral la cual es castaño claro especialmente en la porción anterior, correspondiente a las coxas de las patas. Peines de color amarillo cremoso.

Variaciones. La evaluación de las variaciones de la especie fue realizada con los ejemplares de la Serie Morfométrica (Machos, $\mathrm{n}=17$. A- Sabana de Piedra, Municipio Caripe, Estado Monagas: CELT-318: J. Rondón, 21/ 


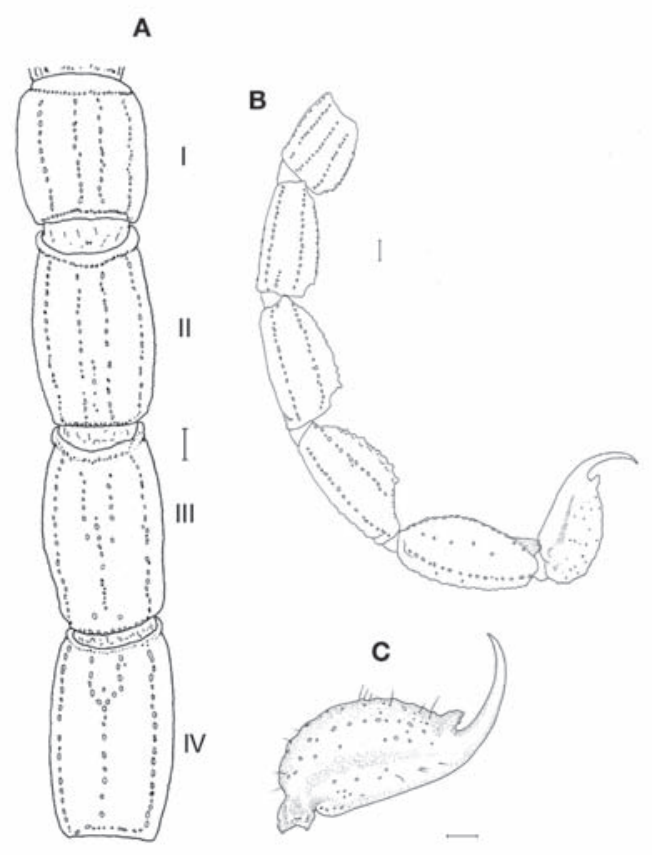

Fig. 5. Tityus quirogae sp. nov. Hembra (CELT-334): a. Metasoma, vista ventral. b. Metasoma, vista lateral. c. Telson, vista lateral. Escala $=1 \mathrm{~mm}$.

Fig. 5. Tityus quirogae sp. nov. Female (CELT-334): a. Metasoma, ventral view. b. Metasoma, lateral view. c. Telson, lateral view. Scale bar $=1 \mathrm{~mm}$.

x/1999. CELT-512 y 513: A. Betancourt, 8/ v/2002 (hacienda Tacarigua). B- La Guanota, Municipio Caripe, Estado Monagas: CELT-405 a 411: E. Asens, 22/ii/2001. CELT-518 y 519: E. Asens, 17/v/2002. C- Serie Tipo: CELT-320, 336, 337 (holotipo), 363 y 400) (Hembras, $n$ = 31. A- Caserío La Loma de Las Trincheras, Municipio Montes, Estado Sucre: CELT-153: L. De Sousa, 20/viii/1998 y CELT-231: E. Marchán-Marcano, 14/viii/1999. B- Sabana de Piedra, Municipio Caripe, Estado Monagas: CELT-179: M. J. Marcano y J. Amundaray, 16/ix/1998 (hacienda Vuelta Larga). CELT251: A. Betancourt, 20/ix/1999 (hacienda Tacarigua, Palma Sola). CELT-317: L. De Sousa, 20/x/1999 (hacienda La Encantada, Vuelta Larga). CELT-319: A. Betancourt, 22/ x/1999 (hacienda La Encantada, Vuelta Larga).

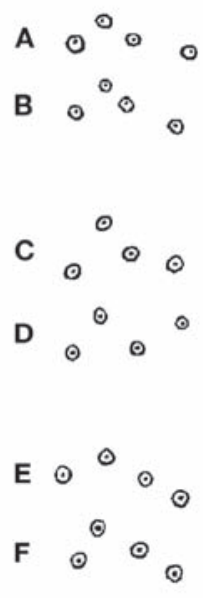

Fig. 6. Comparación del patrón de distribución de las tricobotrias dorsales del fémur de T. meridanus (a: macho, b: hembra), T. nematochirus (c: macho, d: hembra) y $T$. quirogae (e: macho, f: hembra).

Fig. 6. Comparison of the distribution patern of dorsal trichobothria of the femur of T. meridanus (a: male, b: female), T. nematochirus (c: male, d: female) y T. quirogae (e: male, f: female).

CELT-353: A. Betancourt, 22/vi/2000 (hacienda La Encantada, Vuelta Larga). CELT-511: A. Betancourt, 8/v/2002 (hacienda Tacarigua, Palma Sola). C- La Guanota, Municipio Caripe, Estado Monagas: CELT-331: E. Asens, 21/ii/2000. CELT-335: E. Asens, 23/ii/2000. CELT-338 a 341: E. Asens, 20/iii/2000. CELT357 y 358: E. Asens, 17/ix/2000. CELT-412 a 418: E. Asens, 22/ii/2001. CELT-515 a 517: E. Asens, 17/v/2002. D- Serie Tipo: CELT-152, 246, 323, 334 у 399).

Número de dientes pectíneos. Frecuencia en machos $(\mathrm{n}=17,34$ peines, derecho/izquierdo): 18/18 (1), 18/20 (1), 19/18 (1), 19/19 (8), 19/20 (3), 20/19 (2) y 20/20 (1). Frecuencia en hembras $(\mathrm{n}=31,62$ peines, derecho/izquierdo): 17/18 (3), 17/19 (1), 18/17 (1), 18/18 (3), 18/19 (4), 18/20 (1), 19/18 (6), 19/19 (9), 19/20 (1), 20/19 (1) y 20/20 (1).

Número de filas de dentículos del dedo móvil (quela derecha). Frecuencia en machos ( $\mathrm{n}=17,17$ dedos móviles): 15 (2) y 16 (15). 
CUADRO 2

Serie Tipo: Datos morfométricos de los paratipos hembras de Tityus quirogae

TABLE 2

Type Series: Morphometric data of the female paratypes of Tityus quirogae

\begin{tabular}{|c|c|c|c|c|c|c|}
\hline \multicolumn{2}{|l|}{ Región Anatómica } & $\begin{array}{c}\text { CELT-152 } \\
q\end{array}$ & $\begin{array}{c}\text { CELT-246 } \\
q\end{array}$ & $\begin{array}{c}\text { CELT-323 } \\
q\end{array}$ & 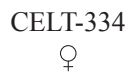 & $\begin{array}{c}\text { CELT-399 } \\
\uparrow\end{array}$ \\
\hline \multicolumn{2}{|l|}{ Longitud caparazón } & 7.90 & 7.80 & 7.34 & 8.27 & 8.60 \\
\hline \multicolumn{2}{|l|}{ Ancho caparazón } & 8.60 & 8.44 & 8.18 & 8.77 & 8.79 \\
\hline \multicolumn{2}{|c|}{ Longitud mano izquierda } & 16.05 & 15.63 & 16.08 & 16.14 & 12.39 \\
\hline \multicolumn{2}{|c|}{ Longitud mano derecha } & 16.14 & 15.61 & 16.10 & 16.09 & 15.42 \\
\hline \multicolumn{2}{|l|}{ Ancho mano derecha } & 2.80 & 2.64 & 2.93 & 3.00 & 2.91 \\
\hline \multicolumn{2}{|c|}{ Longitud dedo móvil (mano derecha) } & 10.07 & 10.53 & 10.38 & 10.34 & 10.74 \\
\hline \multicolumn{2}{|l|}{ Longitud tibia derecha } & 8.35 & 9.22 & 8.93 & 9.61 & 9.88 \\
\hline \multicolumn{2}{|c|}{ Longitud fémur derecho } & 9.05 & 8.72 & 8.83 & 9.20 & 9.31 \\
\hline \multicolumn{2}{|l|}{ Longitud mesosoma } & 25.84 & 22.50 & 22.05 & 26.54 & 23.57 \\
\hline Segmento caudal I: & $\begin{array}{l}\text { Ancho } \\
\text { Largo }\end{array}$ & $\begin{array}{l}4.23 \\
4.86\end{array}$ & $\begin{array}{l}3.79 \\
5.45\end{array}$ & $\begin{array}{l}3.90 \\
5.08\end{array}$ & $\begin{array}{l}4.45 \\
5.86\end{array}$ & $\begin{array}{l}4.26 \\
5.62\end{array}$ \\
\hline Segmento caudal II: & $\begin{array}{l}\text { Ancho } \\
\text { Largo }\end{array}$ & $\begin{array}{l}3.84 \\
6.10\end{array}$ & $\begin{array}{l}3.79 \\
6.85\end{array}$ & $\begin{array}{l}3.89 \\
6.29\end{array}$ & $\begin{array}{l}4.26 \\
6.75\end{array}$ & $\begin{array}{l}4.08 \\
5.06\end{array}$ \\
\hline Segmento caudal III: & $\begin{array}{l}\text { Ancho } \\
\text { Largo }\end{array}$ & $\begin{array}{l}4.04 \\
6.79\end{array}$ & $\begin{array}{l}4.04 \\
8.03\end{array}$ & $\begin{array}{l}3.83 \\
6.62\end{array}$ & $\begin{array}{l}4.26 \\
7.54\end{array}$ & $\begin{array}{l}4.02 \\
7.28\end{array}$ \\
\hline Segmento caudal IV: & $\begin{array}{l}\text { Ancho } \\
\text { Largo }\end{array}$ & $\begin{array}{l}4.13 \\
7.87\end{array}$ & $\begin{array}{l}4.04 \\
8.17\end{array}$ & $\begin{array}{l}4.16 \\
7.91\end{array}$ & $\begin{array}{l}4.63 \\
8.46\end{array}$ & $\begin{array}{l}4.33 \\
7.87\end{array}$ \\
\hline Segmento caudal V: & $\begin{array}{l}\text { Ancho } \\
\text { Largo }\end{array}$ & $\begin{array}{l}4.16 \\
8.04\end{array}$ & $\begin{array}{l}3.85 \\
7.98\end{array}$ & $\begin{array}{l}3.93 \\
8.06\end{array}$ & $\begin{array}{l}4.65 \\
9.50\end{array}$ & $\begin{array}{l}4.29 \\
8.70\end{array}$ \\
\hline \multicolumn{2}{|l|}{ Longitud telson } & 8.58 & 8.47 & 7.99 & 8.12 & 8.33 \\
\hline \multicolumn{2}{|c|}{ Longitud peine derecho } & 4.11 & 4.60 & 4.99 & 4.63 & 4.67 \\
\hline Dientes pectíneos: & $\begin{array}{l}\text { Peine derecho } \\
\text { Peine izquierdo }\end{array}$ & $\begin{array}{l}19 \\
19\end{array}$ & $\begin{array}{l}19 \\
19\end{array}$ & $\begin{array}{l}18 \\
19\end{array}$ & $\begin{array}{l}18 \\
19\end{array}$ & $\begin{array}{l}19 \\
18\end{array}$ \\
\hline \multicolumn{2}{|c|}{ Filas dentículos (dedo móvil derecho) } & 16 & 16 & 16 & 15 & 15 \\
\hline \multicolumn{2}{|l|}{ Longitud total } & 75.98 & 75.25 & 71.34 & 81.04 & 75.03 \\
\hline
\end{tabular}

Todos los datos se expresan en mm (excepto el número de dientes pectíneos y el número de filas de dentículos del dedo móvil derecho). 
Frecuencia en hembras $(\mathrm{n}=31,31$ dedos móviles): 15 (7) y 16 (24).

En el Cuadro 3, se presentan los valores métricos de la Serie Morfométrica de T. quirogae (con sus medianas y límites de confianza al 95\%). Los resultados demuestran que la especie presenta dimorfismo sexual. En las hembras: el ancho y el largo del caparazón, el ancho de la quela derecha, la longitud del mesosoma, el ancho del I al V segmento caudal del metasoma, el largo del telson y la longitud total son significativamente superiores que en los machos. En los machos: los valores del largo de la quela (derecha e izquierda), la tibia, el fémur y el peine derecho son significativamente mayores que en las hembras, por lo que representa la mayor diferencia morfométrica entre ambos sexos.

En algunos escorpiones estudiados $(\mathrm{n}=18$; 11 hembras y 7 machos) se observó alguna de las siguientes alteraciones morfológicas: 1Borde anterior del caparazón con escotadura muy profunda (CELT-319) o borde prácticamente recto (CELT-418). 2- Uno o más dientes pectíneos muy pequeños en comparación con los del peine contralateral (CELT-338, 406, 410 y 517). 3- Alteración en la posición o ubicación de las tricobotrias: $d_{2}$ ubicada en la cara dorsal del fémur acompañando a la $d_{1}, d_{3}, d_{4}$ y $d_{5}$ (CELT-340), ausencia en el dedo fijo de las tricobotrias et (CELT-413), est (CELT-517) y esb (CELT-363) y ausencia de la tricobotria $E b_{3}$ en la base de la quela (CELT-411 y 516). 4- Deformidad traumática de uno o más segmentos del metasoma (CELT-412, 417 y 513).

Distribución e historia natural. Esta nueva especie, hasta el presente, sólo se conoce para los Municipios Caripe (Monagas) y Montes (Sucre) en el tramo oriental de la Cordillera de La Costa (región Nororiental de Venezuela) entre 818 y 1172 msnm (Fig. 7). Habita en el bosque húmedo premontano (bh-P) según la clasificación de zonas de vida (Ewel et al. 1968) y con los tipos de vegetación de bosques ombrófilos submontanos semideciduos estacionales y bosques ombrófilos montanos subsiempreverdes (Huber y Alarcón 1988). El clima de estas áreas es Tipo A, lluvioso cálido (Ami: bosque muy húmedo perennifolio transicional, típico de influencia marítima). Se encuentra, en las plantaciones de café, dentro de los troncos en proceso de descomposición y en las grietas del talud de la carretera. En algunas zonas pobladas (Sabana de Piedra y La Guanota, Municipio Caripe, Estado Monagas) pueden ubicarse intradomiciliarmente en la vivienda humana (CELT-318, 331 y 363). Para dos ejemplares hembras se registró un total de 28 y 32 crías respectivamente.

Juveniles. Los del primer estadio presentan una coloración "atigrada" con manchas pardas sobre un fondo blanco amarillento.

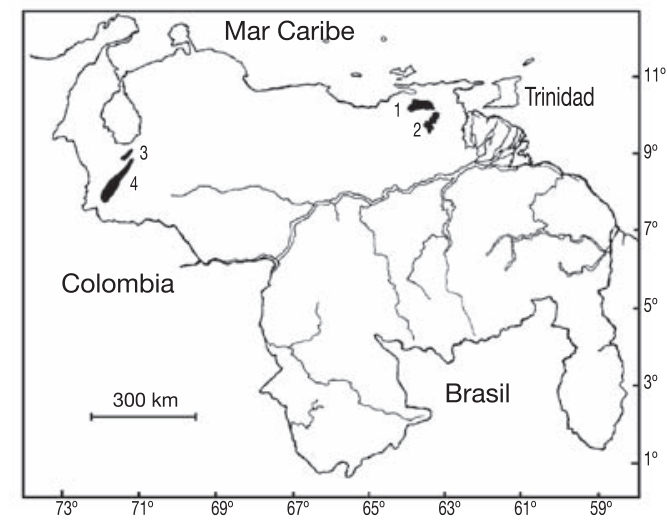

Fig. 7. Distribución de Tityus quirogae sp. nov. en la región Nororiental de Venezuela. Área 1 (Municipio Montes, Estado Sucre) y Área 2 (Municipio Caripe, Estado Monagas). Especies morfológicamente más similares a Tityus quirogae, ubicadas en la región Andina, al occidente de Venezuela: T. meridanus (Área 3: Estado Mérida) y T. nematochirus (Área 4: piedemonte andino, entre los Estados Táchira y Barinas).

Fig. 7. Distribution of Tityus quirogae sp. nov. in the Northeastern region of Venezuela. Area 1 (Montes Municipality, Sucre State) and Area 2 (Caripe Municipality, Monagas State). Species morphologically more similar to Tityus quirogae, located in the Andean region, at the west of Venezuela: T. meridanus (Area 3: Mérida State) and T. nematochirus (Area 4: Andean foothills, between Táchira and Barinas States). 
CUADRO 3

Medianas y límites de confianza al 95\% de los datos morfométricos de ejemplares machos y hembras de Tityus quirogae

TABLE 3

Median and 95\% confidence intervals of the morphometric data of the male and female specimens of Tityus quirogae

\begin{tabular}{|c|c|c|c|c|c|c|}
\hline & \multicolumn{2}{|c|}{$\begin{array}{c}\sigma^{\nearrow} \\
\mathrm{n}=17\end{array}$} & \multicolumn{2}{|c|}{$\begin{array}{c}\stackrel{q}{+} \\
\mathrm{n}=31\end{array}$} & \multirow[b]{2}{*}{$\mathrm{p}=$} \\
\hline & & $\begin{array}{c}\text { Mediana } \\
(\mathrm{mm})\end{array}$ & LC $(95 \%)$ & $\begin{array}{c}\text { Mediana } \\
(\mathrm{mm})\end{array}$ & LC $(95 \%)$ & \\
\hline \multicolumn{2}{|l|}{ Longitud del caparazón } & 7.18 & $6.96-7.42$ & 7.85 & $7.73-7.98$ & 0.00048 \\
\hline \multicolumn{2}{|l|}{ Ancho del caparazón } & 7.79 & $7.62-7.94$ & 8.67 & $8.51-8.84$ & 0.00001 \\
\hline \multicolumn{2}{|c|}{ Longitud de la mano izquierda } & 18.15 & $17.71-19.03$ & 16.19 & $15.91-16.48$ & 0.000001 \\
\hline \multicolumn{2}{|c|}{ Longitud de la mano derecha } & 18.31 & $17.89-18.94$ & 16.15 & $15.88-16.38$ & 0.000001 \\
\hline \multicolumn{2}{|c|}{ Ancho de la mano derecha } & 1.8 & $1.77-1.88$ & 2.89 & $2.84-2.94$ & 0.000001 \\
\hline \multicolumn{2}{|c|}{ Longitud dedo móvil (mano derecha) } & 10.64 & $10.37-11.01$ & 10.48 & $10.29-10.66$ & ns \\
\hline \multicolumn{2}{|c|}{ Longitud de la tibia derecha } & 12.54 & $12.19-12.93$ & 9.66 & $9.43-9.83$ & 0.000001 \\
\hline \multicolumn{2}{|c|}{ Longitud del fémur derecho } & 11.75 & $11.41-12.25$ & 9.13 & $8.99-9.28$ & 0.000001 \\
\hline \multicolumn{2}{|c|}{ Longitud del mesosoma } & 19.06 & $18.55-19.79$ & 23.63 & $22.59-24.74$ & 0.000001 \\
\hline \multirow{2}{*}{ Segmento caudal I: } & Ancho & 3.56 & $3.49-3.65$ & 4.23 & $4.14-4.32$ & 0.000001 \\
\hline & Largo & 5.32 & $5.21-5.50$ & 5.29 & $5.12-5.51$ & ns \\
\hline \multirow{2}{*}{ Segmento caudal II: } & Ancho & 3.30 & $3.22-3.41$ & 3.99 & $3.99-4.06$ & 0.000001 \\
\hline & Largo & 6.62 & $6.50-6.54$ & 6.42 & $6.23-6.62$ & ns \\
\hline \multirow{2}{*}{ Segmento caudal III: } & Ancho & 3.35 & $3.27-3.44$ & 4.02 & $3.93-4.11$ & 0.000001 \\
\hline & Largo & 7.32 & $7.13-7.64$ & 7.16 & $6.93-7.40$ & ns \\
\hline \multirow{2}{*}{ Segmento caudal IV: } & Ancho & 3.60 & $3.53-3.74$ & 4.21 & $4.13-4.31$ & 0.000001 \\
\hline & Largo & 7.93 & $7.75-8.12$ & 7.93 & $7.73-8.15$ & ns \\
\hline \multirow{2}{*}{ Segmento caudal V: } & Ancho & 3.68 & $3.53-3.82$ & 4.19 & $4.08-4.31$ & 0.000002 \\
\hline & Largo & 8.60 & $8.41-8.78$ & 8.74 & $8.51-8.91$ & ns \\
\hline \multicolumn{2}{|l|}{ Longitud del telson } & 8.15 & $7.96-8.29$ & 8.90 & $8.67-9.09$ & 0.00002 \\
\hline \multicolumn{2}{|c|}{ Longitud del peine derecho } & 5.39 & $5.26-5.49$ & 4.63 & $4.54-4.74$ & 0.000001 \\
\hline \multirow{2}{*}{ Dientes pectíneos } & eine derecho & 19 & $19-19$ & 18.5 & $18-19$ & ns \\
\hline & eine izquierdo & 19 & $19-19.50$ & 18.5 & $18.5-19$ & ns \\
\hline \multicolumn{2}{|c|}{ Filas dentículos (dedo móvil derecho) } & 16 & $16-16$ & 16 & $15.50-16$ & ns \\
\hline \multicolumn{2}{|l|}{ Longitud total } & 70.17 & $68.46-72.08$ & 75.61 & $74.09-77.43$ & 0.00079 \\
\hline
\end{tabular}

Todos los datos se expresan en mm (excepto el número de dientes pectíneos y el número de filas de dentículos del dedo móvil derecho), n (número de ejemplares), p (nivel de significación) y ns (no significativo). 
Región ventral del cefalotórax, mesosoma, trocánter y fémur blancos o amarillentos. Acúleo bien definido. Crestas y surcos bien definidos en todo el cuerpo. Segmentos distales de las patas con bandas de color pardo oscuro. El dimorfismo sexual mostrado por los adultos de esta especie no es apreciable en los juveniles del primer estadio.

Etimología. El nombre de la especie, "T. quirogae", es un matronímico en honor a Mercedes Quiroga, pionera de los estudios de la escorpiofauna de la región Nororiental de Venezuela en la Universidad de Oriente, Venezuela.

\section{DISCUSIÓN}

El macho presenta un marcado dimorfismo sexual evidenciado en la forma de los pedipalpos y en la longitud de los peines. $T$. quirogae es la primera especie de su género con pedipalpos delgados y alargados, señalada para la región Nororiental de Venezuela. En este país sólo se conocen, hasta el presente, dos especies con estas características, T. nematochirus y $T$. meridanus (González-Sponga 1981, 1996a, b), distribuidos en la región Andina (en el extremo occidental del país) (Fig. 7). La localidad tipo de $T$. quirogae se encuentra ubicada en una planicie al pie de una montaña con pendientes verticales (superiores al 75\%). En sus alrededores presenta un bosque semideciduo en proceso hacia selva nublada. Esta localidad se encuentra en un territorio de relevancia epidemiológica y clínica, para la cual se han registrado tasas elevadas de morbilidad y mortalidad por envenenamiento escorpiónico (De Sousa et al. 1995, 1997, 1999, 2000). En áreas pobladas, cercanas a la localidad tipo, se localizaron tres ejemplares intradomiciliarmente.

T. quirogae posiblemente sea una especie endémica del tramo oriental de la Cordillera de La Costa venezolana, reforzando los argumentos que caracterizan a la región Nororiental de Venezuela como un área de alto endemismo (Steyermark 1966). En la Cordillera de La Costa, el hecho que la mayoría de las especies endémicas de anfibios se encuentren en las zonas de mayor altitud, sería una evidencia que favorece la estrecha relación entre la abundancia de zonas de vida y la diversidad de la fauna encontrada en ellas (Péfaur y Rivero 2000). Para otras especies de Tityus, en el nororiente venezolano, su hábitat se ubica fundamentalmente en el bosque seco tropical (González-Sponga 1984). Este bosque, situado a menor altitud sobre el nivel del mar, podría actuar como una barrera para el aislamiento de T. quirogae.

La forma y ubicación de las carenas ventrales de los segmentos caudales I al IV en T. quirogae coinciden con los caracteres señalados por González-Sponga (1996a) para el grupo artificial "androcottoides". Sin embargo, la posición y distribución de las tricobotrias así como las semejanzas morfológicas entre la especie descrita y $T$. nematochirus reflejan la afinidad existente entre ambas. Esto apunta hacia la necesidad de una revisión de los grupos "nematochirus" y "androcottoides" sensu Gonzalez-Sponga (1996a) o bien de los caracteres que los definen.

Armas et al. (2002), describen la hembra de T. dedoslargos Francke y Stockwell, 1987, escorpión endémico de Costa Rica (Provincias de San José y Puntarenas). En esta especie "la mayor diferencia morfométrica entre ambos sexos está dada por la gran elongación y esbeltez del pedipalpo en los machos". Habita en bosques húmedos y muy húmedos entre $50 \mathrm{y}$ poco más de $800 \mathrm{msnm}$. T. quirogae presenta la misma característica morfométrica a la descrita por Armas et al. (2002), a lo que se suma el tamaño de los peines en el macho. Al igual que $T$. dedoslargos, habita en bosques húmedos, pero hasta el presente, no se han capturado por debajo de los $700 \mathrm{msnm}$. Según los resultados de Armas et al. (2002), la hembra de $T$. dedoslargos es menos abundante que el macho, lo contrario sucede con T. quirogae. Adicionalmente, desde 1998 hasta el presente, no hemos logrado obtener ejemplares machos de T. quirogae en el Estado Sucre.

Armas et al. (2002), comentan sobre un ejemplar con ambas pectinas anómalas en lo que respecta al número de sus dientes 
pectíneos. En T. quirogae, se observaron alteraciones en las pectinas (cuatro ejemplares) y modificaciones relacionadas con las tricobotrias en lo que respecta a su posición, su ubicación o la ausencia de una de estas estructuras (nueve ejemplares).

Lourenço y Otero Patiño (1998), agrupan las especies de Tityus colombianos en tres grupos: "clathratus", "bahiensis" y "asthenes". El grupo "asthenes" se diagnostica por los siguientes caracteres: longitud total entre 65 y $100 \mathrm{~mm}$, de coloración general negruzca en los adultos y amarillenta manchada de castaño en los inmaduros, tubérculo subaculear espinoide, lámina basal del área intermedia de las pectinas dilatada en casi todas las especies. Estas características indicarían que T. quirogae pertenece al grupo "asthenes". Dentro de este grupo, compuesto por 27 especies (Fet y Lowe 2000, Armas et al. 2002), existen seis cuyos machos presentan pedipalpos marcadamente más largos y delgados que las hembras como característica fundamental relacionada con su dimorfismo sexual: T. asthenes Pocock, 1893; T. nematochirus Mello-Leitão, 1940; T. meridanus González-Sponga, 1981; T. dedoslargos Francke y Stockwell, 1987; T. oteroi Lourenço, 1998 y T. antioquensis Lourenço y Otero Patiño, 1998 a los cuales se suma T. quirogae.

\section{AGRADECIMIENTOS}

Nuestra gratitud a Oscar Leonardo Chinchilla e Ildefonso Liñero (Universidad de Oriente, Núcleo de Sucre, Cumaná) por sus comentarios y la lectura crítica del manuscrito. A María José Marcano, Jelissa Mundaray, Daniela Marín, Luis Alberto Rodríguez Maracay, Rigoberto Valecillos, Ricardo Marcano y Dinora Sánchez por su colaboración en la expediciones de campo en la serranía del Turimiquire del Estado Monagas. A la familia Torrivilla, dueños de la hacienda La Encantada de Sabana de Piedra, Estado Monagas, localidad tipo de Tityus quirogae. A Alcides Betancourt y a la familia Marcano-Marín por su hospitalidad.
A FUNDACITE-Anzoátegui, al Consejo de Investigación UDO y al CDCH-UCV por su apoyo económico. Los dibujos fueron elaborados por Osvaldo Villarreal Manzanilla.

\section{RESUMEN}

Se describe una nueva especie de la familia Buthidae, Tityus quirogae, del Macizo del Turimiquire en el tramo oriental de la Cordillera de La Costa en los Estados Monagas y Sucre, Venezuela. Es similar, morfológicamente, a T. nematochirus y T. meridanus. Se distingue de estas especies por la disposición de las tricobotrias dorsales del fémur y por presentar la siguiente combinación de características: (1) I Segmento caudal con dos carenas ventrales paralelas. Segmentos caudales II al IV con carenas ventrales dobles hacia el área proximal que convergen y se hacen únicas, para finalmente ser divergentes hacia la región distal, (2) marcado dimorfismo sexual, especialmente en la forma de los pedipalpos; las quelas en los machos son marcadamente delgadas y largas, (3) número de dientes pectíneos (macho: 18 derechos y 18 izquierdos y hembra: 18 derechos y 19 izquierdos), (4) número de hileras de dentículos del dedo móvil de la mano derecha (macho 16 y hembra 16). T. quirogae es la primera especie del género con pedipalpos delgados y alargados para la región nororiental de Venezuela.

Palabras clave: escorpión, Buthidae, Tityus, nueva especie, Turimiquire, Venezuela.

\section{REFERENCIAS}

Armas, L.F. de, C. Víquez \& M. Montoya. 2002. Complementos a la descripción de Tityus dedoslargos (Scorpiones: Buthidae). Rev. Biol. Trop. 50: 161-167.

Borges, A. 1996. Escorpionismo en Venezuela. Acta Biol. Venez. 16: 65-75.

De Sousa, L., D. Kiriakos, J. Jiménez, D. Michielli, C. Rodríguez, J. Mirabal \& M. Quiroga. 1995. Accidente cerebrovascular isquémico por emponzoñamiento escorpiónico: observación clínica. SABER (Universidad de Oriente, Venezuela). 7: 7-14.

De Sousa, L., P. Parrilla, L. Tillero, A. Valdiviezo, E. Ledezma, A. Jorquera \& M. Quiroga. 1997. Scorpion poisoning in the Acosta and Caripe counties of Monagas State, Venezuela. Part I: characterization of some epidemiological aspects. Cad. Saúde Públ. 13: 45-51. 
De Sousa, L., S. Bónoli, P. Parrilla-Álvarez, E. Ledezma, A. Jorquera \& M. Quiroga. 1999. The proposal of a new endemic macroregion for scorpionism in Venezuela. J. Venom. Anim. Toxins. 5: 111.

De Sousa, L., P. Parrilla-Álvarez \& M. Quiroga. 2000. An epidemiological review of scorpion stings in Venezuela. The northeastern region. J. Venom. Anim. Toxins. 6: 127-165.

Ewel, J., A. Madriz \& E. Tossi. 1968. Zonas de vida de Venezuela. Ministerio de Agricultura y Cría, Dirección de Investigación, Caracas, Venezuela. 265 p.

Fet, V. \& G. Lowe. 2000. Family Buthidae C. L. Koch, 1837 , p. 54-286. In V. Fet, W.D. Sissom, G. Lowe \& M.E. Braunwalder (eds.). Catalog of the scorpions of the world (1758-1998). New York Entomological Society, Nueva York, EEUU.

Francke, O.F. \& S.A. Stockwell. 1987. Scorpions (Arachnida) from Costa Rica. Special. Publ. Mus. Texas Tech. Univ. 25: 1-24.

González-Sponga, M.A. 1974. Dos nuevas especies de alacranes del género Tityus en las cuevas venezolanas (Scorpionida: Buthidae). Bol. Soc. Venez. Espel. 5: $55-72$.

González-Sponga, M.A. 1981. Seis nuevas especies del género Tityus en Venezuela (Scorpionida: Buthidae). Monog. Cient. Augusto Pi Suñer (Instituto Universitario Pedagógico de Caracas, Venezuela). 12: 3-86.

González-Sponga, M.A. 1984. Escorpiones de Venezuela Cuadernos Lagoven, Caracas, Venezuela. 129 p.

González-Sponga, M.A. 1985. Tres nuevas especies de arácnidos de Venezuela (Scorpionida: Chactidae: Buthidae). Mem. Soc. Cien. Nat. La Salle. 45: 25-45.

González-Sponga, M.A. 1987. Tres nuevas especies del género Tityus de Venezuela (Scorpionida: Buthidae). Bol. Soc. Ven. Cien. Nat. 41: 217-256.

González-Sponga, M.A. 1996a. Guía para identificar escorpiones de Venezuela. Cuadernos Lagoven, Caracas. 204 p.

González-Sponga, M.A. 1996b. Arácnidos de Venezuela: Seis nuevas especies del género Tityus y redescripción de Tityus pococki Hirts, 1907, Tityus rugosus (Schenkel, 1932) n. comb. y Tityus nematochirus Mello-Leitão, 1940 (Scorpionida: Buthidae). Acta Biol. Venez. 16: 1-38.

González-Sponga, M.A. 1997a. Venezuelan arachnides. Two new species of the Tityus genus (Scorpionida:
Buthidae) in the State of Lara. J. Venom. Anim. Toxins. 3: 295-310.

González-Sponga, M.A. 1997b. Arácnidos de Venezuela. Tres nuevas especies de escorpiones de la región amazónica-guayanesa (Buthidae: Chactidae). Soc. Cienc. Nat. La Salle. 57: 55-69.

González-Sponga, M.A. 2001. Arácnidos de Venezuela: cuatro especies nuevas del género Tityus (Scorpionida: Buthidae). Acta Biol. Venez. 21: 69-83.

González-Sponga, M.A., G. D’Suze \& C. Sevcik. 2001 Venezuelan arachnids. Two new species of the Tityus genus (Scorpionida: Buthidae) and the chromatographic profile of venom as a possible taxonomical tool. J. Venom. Anim. Toxins. 7: 219-239.

Huber, O. \& C. Alarcón. 1988. Mapa de Vegetación de Venezuela. Caracas: MARNR. Dirección de Cartografía Nacional. Escala 1:2.000.000, 95 x $70.5 \mathrm{~cm}$.

Lourenço, W.R. 1998. A new species of Tityus C. L. Koch, 1836 (Scorpiones, Buthidae) for Colombia, with a check-list to the Colombian species of the genus Zoosystema 20: 501-511.

Lourenço, W.R. \& R. Otero Patiño. 1998. Tityus antioquensis sp. n., a new species of scorpion from the Department Antioquia, Central Cordillera of Colombia (Scorpiones, Buthidae), with a checklist and key for the Colombian species of the genus. Entomol. Mitt. Zool. Mus. Hamburg. 12: 297-307.

Manzanilla, J., L. De Sousa, M. Quiroga, H. López \& P. Parrilla-Álvarez. 2002. Morfología externa de Rhopalurus laticauda (Scorpiones: Buthidae). SABER (Universidad de Oriente, Venezuela). 14: 94-102.

Mello-Leitão de, C. 1940. Um pedipalpo e dois escorpiões da Colombia. Pap. Avul. Dep. Zool. São Paulo. 1: 51-55.

Mello-Leitão de, C. 1945. Escorpiões Sul-Americanos. Arq. Mus. Nac. (Río de Janeiro, Brasil). 40: 1-468.

Péfaur, J.E. \& J.A. Rivero. 2000. Distribution, speciesrichness, endemism, and conservation of Venezuelan amphibians and reptiles. Amph. Rep. Cons. 2: 42-70.

Pocock, R.I. 1893. A contribution to the study of Neotropical Scorpions. Ann. Mag. Natur. Hist. London. 12: 77-103.

Quiroga, M., L. De Sousa \& P. Parrilla-Álvarez. 2000. The description of Tityus caripitensis. A new Venezuelan scorpion (Scorpionida, Buthidae). J. Venom. Anim. Toxins. 6: 99-117. 
Quiroga, M., L. De Sousa, P. Parrilla-Álvarez \& J. Manzanilla. 2004. The first report of Tityus (Scorpiones: Buthidae) in Anzoátegui State, Venezuela. A new species. J. Venom. Anim. Toxins inclu. Trop. Dis. 10: 10-33.

Scorza, J.V. 1954. Dos nuevas especies de alacranes de Venezuela. Nov. Cient. Mus. Hist. Nat. La Salle, ser. Zool. 12: 1-11.

Sissom, W.D. 1990. Systematics, biogeography, and paleontology, p. 64-160. In G.A. Polis (ed.). The biology of scorpions. Stanford University, Stanford, California, EEUU.
Stahnke, H.L. 1970. Scorpions nomenclature and mesuration. Ent. News. 81: 297-316.

Steyermark, J. 1966. El cerro Turimiquire y la región oriental adyacente. Acta Bot. Venez. 1: 104-168.

Vachon, M. 1974. Êtude des caractères utilisés pour classer les families et les genres de Scorpions (Arachnides). 1. La trichobothriotaxie en arachnologie. Sigles trichobothriaux et types de trichobothriotaxie chez les Scorpions. Bull. Mus. Natn. Hist. Nat. Paris (Sér. 3, n 140), Zool. 104: 857-958. 